\title{
ICRAR Contributions to the Aperture Array Verification Program
}

\author{
P. J. Hall
}

ICRAR/Curtin University, GPO Box U1987, Perth WA 6845, Australia

\begin{abstract}
The new International Centre for Radio Astronomy Research (ICRAR) is a 60M euro joint venture between Curtin University and The University of Western Australia. ICRAR is currently the only non-European collaborator in the Aperture Array Verification Program (AAVP) and will co-lead the AA-lo work package. The proposed work plan emphasizes innovative design for wideband sparse arrays situated at radio-quiet sites, cost-effective manufacturing and deployment methods, and on-site prototyping and characterization. This short paper describes ICRAR, some of its capabilities and the AAVP interaction.
\end{abstract}

\section{Introduction}

The International Centre for Radio Astronomy Research (ICRAR) is a new institute based in Perth, Western Australia. It was launched on September 1, 2009, and is one of three pillars supporting Australia's involvement in the Square Kilometre Array (SKA); the other two major components of the Australian contribution are the Murchison Radioastronomy Observatory (MRO) in WA - one of two short-listed sites for the SKA - and the Australian SKA Pathfinder (ASKAP), a new 36-dish SKA precursor telescope based on Phased Array Feeds and situated at the MRO.

ICRAR is an equal joint venture between Curtin University and The University of Western Australia. Research is conducted at both Curtin and UWA, with the Curtin node favouring radio astronomy engineering and technical astronomy, and the UWA location supporting both astrophysics and information and communications technology (ICT). Funded initially to the extent of 60M euro for five years, ICRAR is expected to be sustainable indefinitely. Along with the two partner universities, the State Government of WA is a major investor, having contributed approximately $13 \mathrm{M}$ euro in cash to the ICRAR venture. Current research staff numbers are about 20, with the complement expected to double in the next two years. Postgraduate students numbers are similar, with the same doubling expected.

Research and development at ICRAR is designed to contribute high impact results to the Australian and international SKA programs. The institute has been formed specifically to advance the SKA and it is therefore not surprising that it is already making significant inputs to activities such as the international SKA preparatory phase (PrepSKA) design study. Inside ICRAR, research and development selected along key themes is managed within four programs:

- science (HI surveys, galaxy formation, variable Universe);

- ICT (data intensive science and techniques, MRO science archive, high performance computing);

- engineering (radio astronomy laboratory, MRO instrumentation, next generation aperture arrays); and

- MRO support (international experiment support, RFI and electromagnetic compatibility, indigenous liaison).
ICRAR's involvement in the European Aperture Array Verification program has grown out of earlier Curtin interests in sparse arrays for mid-band SKA, and from a major role in the Murchison Widefield Array (MWA), an international lowfrequency demonstrator complementing ASKAP on the MRO site.

\section{ICRAR and the Aperture Array Verification Program (AAVP)}

One of the ICRAR's priorities is to contribute new expertise and resources to PrepSKA, with the institute injecting of order 250 person-months in areas ranging from system design (particularly SKA performance and cost simulation), new postprocessing computing architectures, and site characterization and infrastructure (including power) studies. Notwithstanding the breadth of the PrepSKA commitment, fully half of the contributed resources will be via the AAVP, a major component of the PrepSKA Workpackage 2 study.

AAVP will deliver to PrepSKA an approved, verified and costed design for aperture arrays (AAs) in SKA Phase 1, together with a clear development path for Phase 2 AAs. It also provides an umbrella for coordinating European and associated AA developments. Working with Italian (INAF-IRA) partners, ICRAR will co-lead AAVP Workpackage 5, a project designed to deliver a low-band (70-450 MHz) sparse AA design and prototype. This will complement dense AAs (450 - $1000 \mathrm{MHz})$ being developed by other AAVP contributors. In turn, these deliverables - known as AA-lo and AA-hi, respectively - complement other SKA Reference Design technologies being demonstrated by Global SKA institutes.

To accommodate ICRAR's interest in investigating the applicability of sparse AAs beyond $450 \mathrm{MHz}$, ICRAR has defined a slightly broader internal work plan which sees simulation (both array configuration and electromagnetic), design and prototyping extend to above $700 \mathrm{MHz}$.

ICRAR and INAF will take complementary risk paths in WP5, with ICRAR pursuing relatively high-risk developments such as very wideband elements, integrated receivers, low power optical links and, most likely, experimental self-powered antennas based on solar photovoltaic cells and new-technology energy storage devices. First studies have shown that a radio 
quiet site, such as the MRO, is likely to be an enabling factor in making the AA performance - cost ratio attractive for SKA. To examine this hypothesis, ICRAR will construct and install prototype arrays at the MRO, with the AA-lo instrument being of order 500 square metres effective area at $100 \mathrm{MHz}$. ICRAR is also keen to support other prototyping and/or characterization at radio quiet sites, and the expectation is that instruments at the MRO will be accessible to off-shore investigators via internet links, as well as to visiting personnel hosted by ICRAR.

The ICRAR prototyping endeavours will, like those of the other AAVP contributors, be focused strongly on site-ready design and manufacturing. Industry partners will be involved in designing, manufacturing and deploying the demonstration arrays, in order to maximize the reliability of the all- electronic telescope in desert environments such as that of the Murchison.

The overall AAVP structure ensures strong communication between partners, including communication to and from system designers. ICRAR expects to work especially closely with INAF and others in examining the outcomes of existing sparse AA projects, including LOFAR and MWA. Already, ICRAR workers have modelled the MWA in the performance - cost environment of the SKADS cost tool, itself developed as a collaboration between the European sixth-framework SKA Design Study (SKADS), the Australia Telescope National Facility, the SKA Program Development Office, and ICRAR/Curtin.

\section{Conclusions}

ICRAR is a sizeable new institute, formed with the intention of advancing the international SKA project and, through its contribution to the AAVP and collaboration with Europe, it will make a substantial contribution to making aperture arrays attractive for SKA. This complements well Australian work being done in other SKA Reference Design domains, including the new Phased Array Feed developments being pursued by ASKAP. 